\title{
Research on Large Data Storage Technology Based on Cloud Computing Jianying Wang
}

\author{
School of Information Science \& Technology, Zhaotong University, Zhaotong, 65700, China
}

\author{
Keywords: Large Data, Storage Technology, Cloud Computing
}

\begin{abstract}
In the computer network system, due to the continuous expansion of the application of information storage, the information resources of the network are increasing rapidly. The amount of information transmitted through the network continues to grow and a lot of data storage technology becomes more and more important, which has become the key to enterprise information construction. The paper mainly analyzes the large amount of data storage technology based on cloud computing, and focuses on the cloud storage technology. It has a high reference value for the choice of data storage technology in enterprise information construction.
\end{abstract}

\section{Introduction}

With the continuous development of society, computer technology has been rapid development and the computer has been applied to people living in various fields, to provide people with fast and convenient technology. Computer network storage technology plays a very important role in the storage of data. Network data becomes an indispensable component of people's life. Therefore, data storage has become the core of the development of computer network in China. In fact, the rational application of network storage can bring good benefits and results which requires technical staff based on the corresponding storage data on the technology to choose, find the right way to bring the greatest benefits.

\section{The Current Major Computer Data Storage Technology}

In fact, China's computer network storage technology has been widely attention to the community, which also greatly promoted the development of computer network storage technology. The existing computer network storage technology includes several different methods and these methods are based on the characteristics of different data design, which requires the staff to make a reasonable choice, which includes the following.

SAN Technology. SAN technology is when the server's operating system is arranged in a high-performance safe and reliable external storage device, the server can start in the SAN environment, to avoid the built-in disk on the data capacity constraints, but also to avoid The limitations of the built-in disk itself, so as to effectively achieve the system migration and data centralized management, more conducive to the deployment of the work carried out, with a strong server system integration capabilities, SAN technology in the server out of the Built-in disk to bring the limitations and impact, effectively save space, followed by SAN technology can be unified management and scheduling of multiple servers to strengthen the management of the integrity and connectivity. Of course, SAN technology also has some shortcomings, first SAN technology needs to be recommended in a safe, reliable and stable storage system on the process, the process needs to be the storage system and the server can be compatible with each other to avoid reading failure. Second, because of the SAN technology, all servers need to access from the storage device work, at the same time, the computer operating system in the virtual memory also need to access through the SAN, so you can say SAN technology storage device performance requirements Higher. Finally, the most important issue, that is, once the system failure or crash and other issues, the whole system will cause paralysis.

Storage Virtualization Technology. Storage virtualization technology is mainly to store hardware resources, is a collection of virtualization methods, in fact, storage virtualization technology not only covers the storage virtual pool, logical partition, thin provisioning and cluster 
network storage, etc. This kind of virtual storage method effectively analyzes the different actual storage requirements, brings the high level service to the user, and ensures the maximization of the resource utilization of the whole system, which mainly includes symmetrical and asymmetric two ways. Storage virtualization technology can make the network storage system in the network to achieve decentralized, independent. Enhance the overall reliability of the entire system also increased the expansion of resources. This approach helps to improve the competitiveness of core business, mainly due to storage virtualization technology in the storage process to ensure its high stability and high security features, storage virtualization technology performance is high and it can effectively ensure the normal work to carry out to the enterprise to bring more competitive. Virtual storage technology breaks the traditional storage model, the effective combination of memory and external memory. That is using the advantages of physical equipment and broke the limitations of traditional physical equipment, and truly achieves efficient, high performance.

NAS Gateway Technology. In NAS gateways, storage devices typically connect to each other through fiber channel, which enables multiple storage resources connected to the SAN to be accessed and managed through the gateway. NAS gateway technology can manage the way the client uses the document and can deal with the client's request promptly and this kind of processing needs to be established on the basis of the shared agreement. The request of the client is transferred to the gateway, and the gateway translates the request into a block data request to the storage array. When the storage array processes the block data and then passes the result to the gateway, the gateway converts the data into file data to the client. This process ensures transparency, and the whole system becomes flexible and more scalable.

\section{Cloud Computing Related Research}

Cloud computing is an Internet-based computing approach in which shared hardware and software resources and information can be provided to computers and other devices as needed. Cloud is a metaphor of the Internet, the Internet. In the past, the cloud is often used to represent the telecommunications network, and later used to represent the abstraction of the Internet and the underlying infrastructure. Cloud computing is another big change after the big turn of the 1980s to the client-server. Users no longer need to understand the details of the infrastructure in the cloud without having to have the appropriate expertise and no direct control. Cloud computing describes an Internet-based new IT service addition, usage, and delivery model that typically involves the use of the Internet to provide dynamically scalable and often virtualized resources, which means that computing power can also be used as a commodity through the Internet To circulate.

Cloud computing consists of a series of dynamically upgradable and virtualized resources that are shared by all cloud users and can be easily accessed over the network. Users do not need to master the cloud computing technology, only need to be rented by the needs of individuals or groups Cloud computing resources. Cloud computing is another big change after the big turn of the 1980s to the client-server. The emergence of cloud computing is not accidental, as early as the 1960s, McCarthy put forward the computing power as a water and electricity as a public utility to the user's philosophy, which became the origin of cloud computing ideas. In the 20th century, 80's grid computing, 90 years of public computing, the early 21st century virtualization technology, SOA, SaaS application support, cloud computing as a new resource use and delivery model gradually for the academic and industry awareness The China Cloud Development Innovation Industry Alliance evaluation of cloud computing as "information age business model innovation."

Following the personal computer changes, after the Internet changes, cloud computing is seen as the third wave of IT, China's strategic emerging industries is an important part. It will bring life, production and business model of a fundamental change in cloud computing will become the focus of the current society as a whole. Cloud Computing is Distributed Computing, Parallel Computing, Utility Computing, Network Storage Technologies, Virtualization, Load Balancing, and so on. 


\section{Cloud Storage Technology}

According to the different forms of cloud platform deployment, "cloud" can be divided into four types: public cloud, community cloud, mixed cloud and private cloud; the same time, cloud services will be provided through three delivery forms: software as a service (SaaS) Platform as a service (PaaS) and infrastructure as a service (IaaS). Cloud storage is an emerging solution for storing storage resources on the cloud for user access.

Cloud computing is a new concept that extends and derives from the concept of cloud computing, which is the development of Distributed Computing, Parallel Computing and Grid Computing, Through cloud computing technology, network service providers can handle tens of millions of dollars or even hundreds of dollars of information within a few seconds, and "supercomputer" the same powerful network services. Cloud storage concept similar to cloud computing, it is Refers to a cluster of applications, grid technology or distributed file system and other functions, a large number of different types of storage devices in the network through the application software together to work together to provide external data storage and business access functions of a system to ensure data security And save storage space. Users can easily access anytime, anywhere, through any networkable device connected to the cloud.

The Features of Cloud Storage. Just like a cloudy WAN and the Internet, cloud storage for the user is not referring to a specific device, but rather a collection of large amounts of storage devices and servers. Users use cloud storage, not use a storage device, but the use of the entire cloud storage system to bring a kind of data access services. Therefore cloud storage is not storage, but a service. The core of cloud storage is the application software and storage devices, through the application software to achieve the storage device to the storage service changes.

Classification of Cloud Storage. Cloud storage can be divided into three categories: public cloud storage, internal cloud storage, hybrid cloud storage, where public cloud storage can be used as part of private cloud storage and private cloud storage can be deployed in the enterprise data center or the same location facilities. Private clouds can be managed by the IT department of the enterprise or managed by the service provider.

Public cloud storage: As Amazon's Simple Storage Service (S3) and Nutanix provide storage services, they can provide a large number of file storage at low cost. Vendors can keep each customer's storage, applications are independent, Private. More prominent domestic Baidu cloud disk, mobile clouds, cool disk, Huawei network disk, 360 cloud disk, etc

Internal cloud storage: This cloud storage and private cloud storage is similar, the only difference is that it is still located within the corporate firewall. Can provide a private cloud platform: Eucalyptus, 3A Cloud, Lenovo network disk;

Hybrid cloud storage: This cloud storage to the public cloud and private cloud / internal cloud together. It is mainly used for customer access, especially when the need to temporarily configure the capacity of the time from the public cloud to draw a part of the capacity configuring a private or internal cloud is helpful for a rapidly growing load fluctuation or peak.

The Structure of Cloud Storage. The structure of cloud storage system consists of four layers: storage layer, basic management layer, application interface layer and access layer. Cloud storage is SAN bootstrapping technology, storage virtualization, storage logical partition, automatic hierarchical storage, Application performance expansion, thin provisioning and other technologies integrated application.

Storage layer: the storage layer is the basis of cloud storage. Storage device can be FC fiber channel storage devices, NAS and iSCSI and other IP storage devices, it can be SCSI or SAS and other DAS storage devices. The number of large and distributed in different regions, through each other through the wide area network, Internet or FC fiber channel network together. Store storage device is a unified storage device management system, can achieve the storage device logic virtualization management, multi-link redundancy management and hardware device status monitoring and fault maintenance;

Basic management: basic management is the core of cloud storage, cloud storage is the most difficult to achieve part of the basic management through clustering, distributed file system and grid 
computing technology to achieve multiple storage in cloud storage equipment between the work, so that multiple storage devices can provide the same service, and provide more powerful data access performance;

Application interface layer: the application interface is the most flexible part of cloud storage. You can store different application service interfaces according to the actual business type, and provide different application services, such as video monitoring application platform, IPTV and Video on demand application platform, network hard disk reference platform, remote data backup application platform;

Access layer: any authorized user can log in to the cloud storage system through the standard public application interface and enjoy the cloud storage service. Clouding storage operation unit is different, cloud storage provides different types of access and access means. Application storage is a storage device integrates the application software storage device, it not only has the data storage function, also has the application software function, may regard as the server and the storage device Cloud storage system is a multi-storage device, multi-application, multi-service collaborative work of the collection, any single point of the storage system is not cloud storage. There are different storage devices through the cluster technology, distributed file system and Grid computing and other technologies to achieve the cooperation between multiple storage devices and multiple storage devices can provide the same service, to provide more powerful data access performance.

\section{Conclusion}

With the continuous development and application of large number of data storage technologies in our country, the advantages of various technologies are revealed, and only the analysis and utilization of these technologies can be used to ensure the efficient implementation of storage technology. This requires the relevant staff to conduct a more professional analysis and research to strengthen the applications of the large data storage technology based on cloud computing and promote the development of a large number of computer data storage technology.

\section{References}

[1] Wang Gang. Computer network storage technology[J]. Journal of Computer Applications, 2015 (1): $14-20$.

[2] Han Xudong. Analysis of computer networks in the common storage technology [J]. Information and communication, 2015 (2): 164.

[3] Huang Jinuo, Liu Tao. Analysis of some common computer network storage technology[J]. Information Systems Engineering, 2012 (7): 18-19.

[4] Wang Gang. Computer network storage technology[J]. Journal of Computer Applications, 2015, 24 (1): 14-20.

[5] He Fengru. Network storage mainstream technology and its development trend[J] .Guangdong Radio and Television University, 2009 (2). 